\title{
A Prognostic Nomogram and Heat Map to Predict Survival in Stage II/III Gastric Cancer Patients After Curative Gastrectomy Followed by Adjuvant Chemotherapy
}

\author{
Litong Shi, Zehua Wang (D), Lei Wang, Yongxu Jia, Jing Li, Yanru Qin \\ Department of Oncology, First Affiliated Hospital of Zhengzhou University, Zhengzhou, Henan, People's Republic of China \\ Correspondence: Yanru Qin, Department of Oncology, Zhengzhou University First Affiliated Hospital, No. I Jianshe East Road, Erqi District, \\ Zhengzhou, 450052, Henan, People's Republic of China, Tel +86 I3676932999, Email yanruqin@I63.com
}

\begin{abstract}
Purpose: This study aimed to study the prognostic value of clinicopathological data, inflammation and nutritional indicators, and to design an effective prognostic nomogram and heat map to predict cancer-specific survival (CSS) and disease-free survival (DFS) of stage II/III GC patients who underwent curative gastrectomy with adjuvant chemotherapy.

Patients and Methods: We retrospectively analyzed the data of 611 patients with stage II/III GC after curative gastrectomy followed by adjuvant chemotherapy from $3 \mathrm{GC}$ disease centers. Patients were divided into a training cohort $(\mathrm{n}=503)$ and an external validation cohort $(n=108)$. Nomograms were established based on independent predictors identified by Cox regression analysis in the training cohort. The consistency index (C-index) and the calibration curve were used to evaluate the discriminative ability and accuracy of the nomogram. Heat maps were constructed with the prognostic factors and the corresponding survival probability. We further divided the patients into low-risk and high-risk groups based on the risk score of the nomogram.

Results: Through univariate and multivariate survival analysis, the independent risk factors common to CSS and DFS were identified. Then these predictors were incorporated into the nomograms, and the established nomograms used to predict CSS and DFS had high discriminative power in the training cohort. Meanwhile, the calibration curves of CSS and DFS probability also showed good agreement between the prediction based on the nomograms and the actual observation results. The above independent predictors were applied to establish heat maps. Compared with low-risk patients, the high-risk patients calculated according to the nomogram had a shorter survival time and a worse prognosis.

Conclusion: We established a nomogram and heat map, which could be used to assess the survival rate of stage II/III GC patients who underwent curative gastrectomy with adjuvant chemotherapy. These tools had high prognostic prediction accuracy and provided inspiration for clinical decision-making.
\end{abstract}

Keywords: heat map, nomogram, gastric cancer, adjuvant chemotherapy, prognosis, survival

\section{Introduction}

Gastric cancer (GC) is the fourth most common malignant tumor in the world and the second leading cause of cancer-related deaths, with the highest incidence in the geographic distribution of East Asia, including China. ${ }^{1,2}$ Due to the lack of typical clinical symptoms of early GC, the diagnosis rate of early GC is very low as a large number of GC patients are diagnosed at the advanced or metastatic stage. ${ }^{3}$ At present, the main treatment for GC is curative gastrectomy supplemented by adjuvant chemotherapy or radiotherapy. However, the overall survival rate is still poor even after comprehensive treatment due to recurrence, metastasis and other factors. ${ }^{4,5}$ This requires us to individually analyze independent risk factors and make corresponding risk stratification to improve prognosis prediction, which will also be a new trend in the field of GC research.

A large number of studies have reported that many inflammatory and nutritional factors are related to the prognosis of patients with GC. Indicators such as lymphocyte-monocyte ratio (LMR), neutrophil-lymphocyte ratio 
(NLR), platelet-lymphocyte ratio (PLR), albumin-fibrinogen ratio (AFR) and aspartate aminotransferase (AST)-alanine aminotransferase (ALT) ratio (SLR) were reported to be associated with the prognosis of many types of cancer, including GC. ${ }^{6-11}$ Standardizing these indicators can further accurately evaluate prognosis and improve clinical utility.

So far, the widely used staging method for GC is still the TNM classification system of the eighth edition of the American Joint Committee on Cancer (AJCC). However, the lack of clinicopathological factors and laboratory indicators in the TNM classification system makes it applicable to the general population rather than individual patients. As a new method of quantifying cancer risk, nomogram displays important factors for prognosis and provides more accurate survival predictions. There have been some established nomograms that can predict the survival rate of patients with GC. The 6-variable predictive model for patients with stage II/III GC constructed by Han et al showed that its predictive value was significantly better than that of AJCC-TNM staging system. ${ }^{12}$ However, its predictive power for recurrence was not evaluated. Jiang et al introduced a nomogram of the association of adjuvant chemotherapy with survival in patients with stage II /III GC, but its consistency index (C-index) was only $0.686 .{ }^{13}$ There have been also prognostic models of GC developed based on individual serum markers but lacking external validation. ${ }^{11}$ In addition, GC-related nomograms in previous studies usually had only limited research variables.

This study therefore tried to establish a more comprehensive nomogram and heat map to improve the survival prediction of stage II/III GC patients with adjuvant chemotherapy and facilitate clinicians to identify high-risk patients and guide follow-up treatment.

\section{Patients and Methods}

\section{Patients and Data Collection}

We collected 503 patients with stage II/III GC after curative gastrectomy followed by adjuvant chemotherapy in the First Affiliated Hospital of Zhengzhou University and Henan Cancer Hospital from September 2012 to December 2018 as the training cohort. According to the same standard, the data of 108 patients from April 2014 to November 2017 from the Henan Provincial People's Hospital were collected as the external validation cohort.

Inclusion criteria: (1) stage II/III GC confirmed by histological pathology; (2) negative surgical margin (R0); (3) adjuvant chemotherapy after curative gastrectomy; (4) no preoperative anticancer treatments; (5) complete clinicopathological and follow-up data (all biomarkers were measured within 1 week before surgery); (6) no extraintestinal nutrition, acute inflammation or severe organ damage within 1 week before surgery; (7) no other malignant tumors, no cause of death other than GC. Exclusion criteria: (1) merging other systemic tumors; (2) lacking or incomplete clinical data; (3) receiving neoadjuvant chemotherapy or preoperative radiotherapy. Tumor stage was reclassified according to the AJCC 8th TNM classification.

We grouped common clinical variables such as hemoglobin (HGB), platelets (PLT), albumin (ALB), fibrinogen (FIB), carcinoembryonic antigen (CEA), carbohydrate antigen 19-9 (CA19-9) and CA72-4 by widely accepted thresholds. The best cut-off values for age, adjuvant chemotherapy cycles, tumor size, LMR, NLR, PLR, AFR and SLR were determined by X-tile analysis because of the lack of thresholds.

The informed consent statements of patients could not be obtained because the study was a retrospective analysis. The procedure for this study was reviewed and approved by the Ethics Committee of Zhengzhou University. The study complied with the standards of the Declaration of Helsinki, and patient data were anonymous and strictly confidential.

\section{Follow-Up and Outcome}

Follow-up methods included medical records and telephone investigation. Patients were observed after treatment at 3-month intervals during the first year, every 6 months for 2 to 3 years, and once a year thereafter. Each follow-up included physical examination, laboratory examination, electronic gastroscopy, chest/abdomen/pelvic enhanced CT. Cancer recurrence or metastasis could be diagnosed when local recurrence or distant metastasis was found for the first time after the operation was completed, which was confirmed based on imaging studies or biopsy. The latest follow-up date was October 2021. The primary endpoint of the study was cancer-specific survival (CSS). CSS was defined as 
calculated from the date of surgery until cancer-related death or the last follow-up. Disease-free survival (DFS) was the time from the date of surgery to the date of recurrence/metastasis or the final follow-up.

\section{Statistical Analysis}

The data were analyzed by SPSS Statistics (Version 26.0, IBM, USA), and the clinical characteristics were described by percentage, median, and interquartile (IQR). Categorical variables were compared using the Chi-squared test or Fisher's exact test. Two-tailed $\mathrm{P} \leq 0.05$ was considered statistically significant. The significant variables of univariate analysis were included in Cox multivariate analysis to determine the independent factors. On the basis of the final multivariate Cox model, the rms and survival packages in R (version 4.1.1, Austria, http://www.r-project.org) were used to construct nomograms.

External validation cohort was carried out to prove the reliability and repeatability of the nomograms. The C-index was used to evaluate the prediction effect of the nomograms. Its maximum value was 1 , which meant $100 \%$ prediction effect, and the minimum value was 0.5 . Bootstraps with 1000 resamples was used to draw calibration curves to evaluate the fitting degree of the nomograms. The risk scores were calculated for each patient based on the clinical indicators. The formula was as follows:

$$
\text { Risk score }=\sum_{i=1}^{n} \text { coef clinical indicators }
$$

Patients were divided into low-risk and high-risk groups based on the median risk score. Kaplan-Meier method was used to draw CSS and DFS survival curves, and Log rank test was used for statistical analysis. In addition, we also made heat maps based on the Cox models and the nomograms to show the relationship between the independent predictors and the corresponding survival probabilities.

\section{Results}

\section{Clinicopathological Characteristics of Patients}

The detailed clinicopathological characteristics of 611 patients are provided in Table 1, including 503 patients in the training cohort and 108 patients in the external validation cohort. Among the training cohort cases, the diagnosis age was 28 to 83 years old, and the median age was 59 years old (IQR, 48 to 65 years old). There were 373 males (74.2\%) and 130 females (25.8\%). Adenocarcinoma was the most common pathological type (488, 97.0\%). Poor differentiation (348, $69.2 \%$ ) was the most common tumor grade. The median tumor size was $4.0 \mathrm{~cm}$ (IQR, 3.0-6.0 cm). Only $82(16.3 \%)$ patients showed positive HER-2. According to the AJCC 8th TNM classification, most tumors were classified into PT3 4 stages $(338,67.2 \%)$, lymph node invasion was common (311, 61.8\%), and most were stage III $(303,60.2 \%)$. In addition, 227 patients $(45.1 \%)$ received more than 4 cycles of adjuvant chemotherapy, and the remaining patients only received 1 to 4 cycles. 373 (74.2\%) patients chose the platinum-based doublet regimen. There were 79 patients (15.7\%) who chose taxanes contained regimen, and the remaining patients received monotherapy $(31,6.2 \%)$ or anthracycline-based regimen $(20,4.0 \%)$. The median age of the external validation cohort was 57 years old (IQR, 47 to 67 years old) and included a lower proportion of male patients $(67.6 \%$ versus $74.3 \%)$. Overall, the baseline characteristics were balanced between the training and the external validation cohorts.

The median follow-up time for all patients was 37.0 months (range, 0.9-94.2 months). In the training cohort, the CSS rates of 1-, 3-, and 5-year were $90.4 \%, 65.9 \%$, and $60.3 \%$, respectively. The 1-, 3-, and 5-year DFS rates were $75.5 \%$, $52.1 \%$, and $46.6 \%$, respectively. In the external validation cohort, the CSS rates of 1-, 3-, and 5-year were 94.0\%, $67.9 \%$, and $59.7 \%$, respectively. The 1 -, 3 -, and 5-year DFS rates were $81.5 \%, 61.5 \%$, and $55.8 \%$, respectively.

\section{Independent Prognostic Factors}

The Cox proportional hazards regression models were selected by us to analyze the independent prognostic factors of CSS and DFS. The results of univariate analysis are shown in Table 2. In univariate analysis, age, blood type, tumor size, tumor differentiation, depth of invasion, lymph node invasion, HER-2, adjuvant chemotherapy regimen, adjuvant chemotherapy cycles, HGB, ALB, CA19-9, CA72-4, AFR and SLR were considered to be related to CSS (P $\leq 0.05$ ), 
Table I Baseline Characteristics of Patients in the Training and Validation Cohorts

\begin{tabular}{|c|c|c|c|}
\hline \multirow[t]{2}{*}{ Variables } & \multicolumn{2}{|c|}{ No. (\%) } & \multirow[t]{2}{*}{$\mathbf{P}$} \\
\hline & Training Cohort & Validation Cohort & \\
\hline \multicolumn{4}{|l|}{ Clinical factors } \\
\hline Age, years, median (IQR) & $59(48,65)$ & $57(47,67)$ & 0.700 \\
\hline$\leq 60$ & $285(56.7)$ & $59(54.6)$ & \\
\hline$>60$ & $218(43.3)$ & $49(45.4)$ & \\
\hline Sex & & & 0.163 \\
\hline Male & $373(74.2)$ & $73(67.6)$ & \\
\hline Female & $130(25.8)$ & $35(32.4)$ & \\
\hline Family history & & & $0.7 \mid 4$ \\
\hline No & $38 \mathrm{I}(75.7)$ & $80(74.1)$ & \\
\hline Yes & $122(24.3)$ & $28(25.9)$ & \\
\hline Diabetes & & & 0.166 \\
\hline No & $467(92.8)$ & $96(88.9)$ & \\
\hline Yes & $36(7.2)$ & $12(11.1)$ & \\
\hline Hypertension & & & 0.292 \\
\hline No & $417(82.9)$ & $94(87.0)$ & \\
\hline Yes & $86(17.1)$ & $14(13.0)$ & \\
\hline Thrombus & & & 0.917 \\
\hline No & $469(93.2)$ & $101(93.5)$ & \\
\hline Yes & $34(6.8)$ & $7(6.5)$ & \\
\hline Tobacco & & & 0.142 \\
\hline No & $35 I(69.8)$ & $83(76.9)$ & \\
\hline Yes & $152(30.2)$ & $25(23.1)$ & \\
\hline Alcohol & & & 0.213 \\
\hline No & 387 (76.9) & 77 (7I.3) & \\
\hline Yes & $116(23.1)$ & $31(28.7)$ & \\
\hline Blood type & & & $<0.001$ \\
\hline Type A & $173(34.4)$ & $32(29.6)$ & \\
\hline Type B & $135(26.8)$ & $29(26.9)$ & \\
\hline Type AB & 59 (11.7) & $15(13.0)$ & \\
\hline Type O & $136(27.0)$ & $32(29.6)$ & \\
\hline ACT regimen & & & 0.002 \\
\hline Platinum-based doublet regimen & $373(74.2)$ & $75(69.4)$ & \\
\hline Taxanes contained regimen & $79(15.7)$ & $21(19.4)$ & \\
\hline Monotherapy & $31(6.2)$ & $7(6.5)$ & \\
\hline Anthracycline-based regimen & $20(4.0)$ & $5(4.6)$ & \\
\hline ACT cycles, median (IQR) & $4(3,6)$ & $5(3,7)$ & 0.692 \\
\hline$<5$ & $276(54.9)$ & $57(52.8)$ & \\
\hline$\geq 5$ & $227(45.1)$ & $51(47.2)$ & \\
\hline \multicolumn{4}{|l|}{ Pathological factors } \\
\hline Tumor location & & & $<0.001$ \\
\hline Upper I/3 & $224(44.5)$ & $36(33.3)$ & \\
\hline Middle I/3 & $91(18.1)$ & 31 (28.7) & \\
\hline
\end{tabular}

(Continued) 
Table I (Continued).

\begin{tabular}{|c|c|c|c|}
\hline \multirow[t]{2}{*}{ Variables } & \multicolumn{2}{|c|}{ No. (\%) } & \multirow[t]{2}{*}{$\mathbf{P}$} \\
\hline & Training Cohort & Validation Cohort & \\
\hline Lower $1 / 3$ & $160(31.8)$ & $35(32.4)$ & \\
\hline Mix & $28(5.6)$ & $6(5.6)$ & \\
\hline Tumor size, cm, median (IQR) & $4(3,6)$ & $4(3,6)$ & 0.984 \\
\hline$<4.5$ & $266(52.9)$ & $57(52.8)$ & \\
\hline$\geq 4.5$ & $237(47.1)$ & $51(47.2)$ & \\
\hline Tumor differentiation & & & 0.892 \\
\hline Poor & $348(69.2)$ & $74(68.5)$ & \\
\hline High & $155(30.8)$ & $34(31.5)$ & \\
\hline Depth of invasion & & & 0.078 \\
\hline TI-2 & $165(32.8)$ & $45(4 I .7)$ & \\
\hline T3-4 & $338(67.2)$ & $639(58.3)$ & \\
\hline Lymph node invasion & & & 0.346 \\
\hline No & $192(38.2)$ & $36(33.3)$ & \\
\hline $\mathrm{NI}-3$ & $311(61.8)$ & $72(66.7)$ & \\
\hline Pathology & & & $<0.001$ \\
\hline ADC & $488(97.0)$ & $104(96.3)$ & \\
\hline SCC & $10(2.0)$ & $2(1.9)$ & \\
\hline MANEC & $5(1.0)$ & $2(1.9)$ & \\
\hline HER-2 & & & 0.533 \\
\hline Negative & $421(83.7)$ & $93(86.1)$ & \\
\hline Positive & $82(16.3)$ & $15(13.9)$ & \\
\hline \multicolumn{4}{|l|}{ Laboratory factors } \\
\hline HGB, g/L, median (IQR) & $126.00(107.00,139.00)$ & $125.00(104.25,137.75)$ & 0.477 \\
\hline$<110 / 120$ & $162(32.2)$ & $3 \mid(28.7)$ & \\
\hline$\geq 110 / 120$ & $34 I(67.8)$ & 77 (7I.3) & \\
\hline PLT, $\times 10^{9} / \mathrm{L}$, median (IQR) & $214.00(172.00,252.00)$ & $193.00(156.50,241.00)$ & 0.753 \\
\hline$<350$ & 47 I $(93.6)$ & $102(94.4)$ & \\
\hline$\geq 350$ & $32(6.4)$ & $6(5.6)$ & \\
\hline ALB, g/L, median (IQR) & $32.50(30.00,35.60)$ & $31.50(29.23,36.28)$ & 0.197 \\
\hline$<35$ & $375(74.6)$ & $74(68.5)$ & \\
\hline$\geq 35$ & $128(25.4)$ & $34(31.5)$ & \\
\hline FIB, g/L, median (IQR) & $2.97(2.54,3.47)$ & $2.70(2.50,3.33)$ & 0.411 \\
\hline$\leq 4$ & $469(93.2)$ & $103(95.4)$ & \\
\hline$>4$ & $34(6.8)$ & $5(4.6)$ & \\
\hline CEA, ng/mL, median (IQR) & $1.95(1.11,4.70)$ & $1.69(0.97,3.98)$ & 0.264 \\
\hline$\leq 5$ & $390(77.5)$ & $89(82.4)$ & \\
\hline$>5$ & $113(22.5)$ & $19(17.6)$ & \\
\hline CAI99, U/mL, median (IQR) & $9.14(5.04,20.73)$ & $8.09(4.88,24.25)$ & 0.353 \\
\hline$\leq 37$ & $415(82.5)$ & $85(78.7)$ & \\
\hline$>37$ & $88(17.5)$ & $23(21.3)$ & \\
\hline
\end{tabular}

(Continued) 
Table I (Continued).

\begin{tabular}{|c|c|c|c|}
\hline \multirow[t]{2}{*}{ Variables } & \multicolumn{2}{|c|}{ No. (\%) } & \multirow[t]{2}{*}{$\mathbf{P}$} \\
\hline & Training Cohort & Validation Cohort & \\
\hline CA724, U/mL, median (IQR) & $1.92(\mathrm{I} . \mathrm{II}, 4.48)$ & I.7I $(1.22,3.88)$ & 0.466 \\
\hline$\leq 6.9$ & $439(87.3)$ & 97 (89.8) & \\
\hline$>6.9$ & $64(12.7)$ & II (10.2) & \\
\hline LMR, median (IQR) & $4.00(3.00,5.09)$ & $4.00(2.86,4.78)$ & 0.482 \\
\hline$<4.71$ & $346(68.8)$ & $78(72.2)$ & \\
\hline$\geq 4.71$ & $157(3 \mid .2)$ & $30(27.8)$ & \\
\hline NLR, median (IQR) & $1.76(1.20,2.55)$ & $1.90(1.37,2.53)$ & 0.257 \\
\hline$<1.90$ & $277(55.1)$ & $53(40.1)$ & \\
\hline$\geq 1.90$ & $226(44.9)$ & $55(50.9)$ & \\
\hline PLR, median (IQR) & $132.00(100.77,|7| . \mid 8)$ & $125.58(100.95,162.57)$ & 0.315 \\
\hline$<134.46$ & $262(52.1)$ & $62(57.4)$ & \\
\hline$\geq 134.46$ & $24 I(47.9)$ & $46(42.6)$ & \\
\hline AFR, median (IQR) & $10.79(9.17,13.43)$ & $|2.3|(9.22, \mid 4.26)$ & 0.164 \\
\hline$<16.07$ & $448(89.1)$ & $101(93.5)$ & \\
\hline$\geq 16.07$ & $55(10.9)$ & $7(6.5)$ & \\
\hline SLR, median (IQR) & $1.29(1.05,1.57)$ & $1.34(1.01,1.64)$ & 0.259 \\
\hline$<1.28$ & $249(49.5)$ & $47(43.5)$ & \\
\hline$\geq 1.28$ & $254(50.5)$ & $61(56.5)$ & \\
\hline
\end{tabular}

Abbreviations: iQR, interquartile; ACT, adjuvant chemotherapy; ADC, adenocarcinoma; SCC, squamous cell carcinoma; MANEC, mixed adenoneuroendocrine carcinoma; HGB, Hemoglobin; PLT, Platelets; ALB, albumin; FIB, fibrinogen; CEA, carcinoembryonic antigen; CA, carbohydrate antigen; LMR, lymphocyte-monocyte ratio; NLR, neutrophil-lymphocyte ratio; PLR, platelet-lymphocyte ratio; AFR, albumin-fibrinogen ratio; SLR, aspartate aminotransferase (AST)-alanine aminotransferase (ALT) ratio.

see Table 2 for details. For DFS, similar results were shown in univariate analysis, but blood type was irrelevant (Table 3). Among them, age $(\mathrm{P}<0.001)$, blood type $(\mathrm{P}=0.002)$, adjuvant chemotherapy regimen $(\mathrm{P}<0.001)$, adjuvant chemotherapy cycles $(\mathrm{P}<0.001)$, tumor size $(\mathrm{P}<0.001)$, tumor differentiation $(\mathrm{P}=0.007)$, depth of invasion $(\mathrm{P}<$ 0.001), lymph node invasion $(\mathrm{P}<0.001)$, HER-2 $(\mathrm{P}=0.006)$, AFR $(\mathrm{P}=0.014)$ and SLR $(\mathrm{P}=0.023)$ were further determined by multivariate analysis to be independent prognostic factors of CSS (Table 2). And age $(\mathrm{P}<0.001)$, adjuvant chemotherapy regimen $(\mathrm{P}<0.001)$, adjuvant chemotherapy cycles $(\mathrm{P}<0.001)$, tumor size $(\mathrm{P}<0.001)$, tumor differentiation ( $\mathrm{P}=0.001)$, depth of invasion $(\mathrm{P}<0.001)$, lymph node invasion $(\mathrm{P}<0.001)$, HER-2 $(\mathrm{P}<0.001)$ and ALB $(\mathrm{P}=0.011)$ were independent prognostic factors of DFS. The hazard ratio (HR) and $95 \%$ confidence interval $(\mathrm{CI})$ of the variables are shown in Tables 2 and 3.

\section{Establishment and Validation of the Nomograms}

We tried to establish prognostic nomograms based on the results of the above multivariate analysis after excluding interventional clinical features including adjuvant chemotherapy regimens and cycles (Figure 1). The models could estimate the corresponding 3-, and 5-year CSS and DFS from the weighted total score calculated for each variable. The lower the total score, the better the clinical prognosis. In the internal validation of the prognostic nomograms, the results of the bootstrap method showed that the C-index for predicting CSS and DFS were 0.774 (95\% CI: 0.743-0.805) and 0.713 (95\% CI: $0.682-0.744)$, indicating the nomograms had good discrimination. In order to further evaluate the prediction accuracy of the nomograms, we also drew calibration curves (Figure 2A-D), where the drawn straight line was close to the diagonal, indicating that the prediction of the nomograms fitted well with the actual situation observed, that was, the prediction was close reality. In the external validation cohort, the C-index for predicting CSS and DFS were 0.788 (95\% CI: $0.719-0.857$ ) and 0.727 (95\% CI: 0.656-0.798). The optimal agreement could also be observed in the calibration curves (Figure 2E-H). 
Table 2 Univariate and Multivariate Cox Regression Analyses of Prognostic Factors for Cancer-Specific Survival

\begin{tabular}{|c|c|c|c|c|c|c|}
\hline \multirow[t]{2}{*}{ Variables } & \multicolumn{3}{|c|}{ Univariate Analysis } & \multicolumn{3}{|c|}{ Multivariate Analysis } \\
\hline & HR & $95 \% \mathrm{Cl}$ & $\mathbf{P}$ & HR & $95 \% \mathrm{Cl}$ & $\mathbf{P}$ \\
\hline Age $(>60$ versus $\leq 60)$ & 2.930 & $2.144-4.004$ & $<0.001$ & 2.097 & $1.472-2.988$ & $<0.001$ \\
\hline Sex (male versus female) & 1.183 & $0.824-1.698$ & 0.364 & & & \\
\hline Family history (yes versus no) & 0.815 & $0.562-1.184$ & 0.284 & & & \\
\hline Diabetes (yes versus no) & 0.665 & $0.340-1.303$ & 0.235 & & & \\
\hline Hypertension (yes versus no) & 0.810 & $0.536-1.223$ & 0.317 & & & \\
\hline Thrombus (yes versus no) & 0.831 & $0.439-1.575$ & 0.571 & & & \\
\hline Tobacco (yes versus no) & 1.327 & $0.965-1.825$ & 0.082 & & & \\
\hline Alcohol (yes versus no) & 1.105 & $0.78 \mathrm{I}-1.564$ & 0.571 & & & \\
\hline Blood type & & & 0.012 & & & 0.002 \\
\hline Type A & 1 & & & 1 & & \\
\hline Type B & 1.596 & $1.065-2.392$ & 0.023 & 1.783 & $1.172-2.7 \mid 4$ & 0.007 \\
\hline Type AB & 1.012 & $0.640-1.602$ & 0.958 & 1.212 & $0.737-1.993$ & 0.448 \\
\hline Type O & 1.880 & $1.135-3.115$ & 0.014 & 2.472 & $1.45 \mathrm{I}-4.210$ & 0.001 \\
\hline $\mathrm{ACT}$ regimen & & & $<0.001$ & & & $<0.001$ \\
\hline Platinum-based doublet regimen & 1 & & & 1 & & \\
\hline Taxanes contained regimen & 0.105 & $0.057-0.195$ & $<0.001$ & 0.134 & $0.066-0.274$ & $<0.001$ \\
\hline Monotherapy & 0.155 & $0.079-0.303$ & $<0.001$ & 0.263 & $0.120-0.578$ & 0.001 \\
\hline Anthracycline-based regimen & 0.237 & $0.112-0.501$ & $<0.001$ & 0.198 & $0.084-0.470$ & $<0.001$ \\
\hline ACT cycles $(<5$ versus $\geq 5)$ & 2.812 & $2.021-3.913$ & $<0.001$ & 2.179 & $1.505-3.154$ & $<0.001$ \\
\hline Tumor location & & & 0.455 & & & \\
\hline Upper I/3 & I & & & & & \\
\hline Middle I/3 & 1.064 & $0.5 \mid 3-2.207$ & 0.868 & & & \\
\hline Lower $1 / 3$ & 0.821 & $0.372-1.814$ & 0.626 & & & \\
\hline Mix & 1.200 & $0.573-2.513$ & 0.629 & & & \\
\hline Tumor size $(\geq 4.5 \mathrm{~cm}$ versus $<4.5 \mathrm{~cm})$ & 1.933 & $1.422-2.628$ & $<0.001$ & 2.201 & $1.546-3.135$ & $<0.001$ \\
\hline Differentiation (poor versus high) & 2.193 & $1.507-3.190$ & $<0.001$ & 1.725 & $1.158-2.569$ & 0.007 \\
\hline Depth of invasion (T3-4 versus TI-2) & 1.778 & $1.252-2.524$ & 0.001 & 2.896 & $1.912-4.387$ & $<0.001$ \\
\hline Lymph node invasion (NI-3 versus N0) & 1.827 & $1.309-2.550$ & $<0.001$ & 3.151 & $2.089-4.754$ & $<0.001$ \\
\hline \multicolumn{7}{|l|}{ Pathology } \\
\hline ADC & 1 & & & & & \\
\hline SCC & 0.954 & $0.133-6.844$ & 0.963 & & & \\
\hline MANEC & 2.575 & $0.309-21.480$ & 0.382 & & & \\
\hline HER-2 (positive versus negative) & 1.621 & $1.085-2.423$ & 0.018 & 1.820 & I.189-2.785 & 0.006 \\
\hline HGB $(<110 / 120 \mathrm{~g} / \mathrm{L}$ versus $\geq 110 / 120 \mathrm{~g} / \mathrm{L})$ & 1.489 & $1.092-2.029$ & 0.012 & 0.950 & $0.678-1.332$ & 0.767 \\
\hline PLT $\left(\geq 350 \times 10^{9} / \mathrm{L}\right.$ versus $\left.<350 \times 10^{9} / \mathrm{L}\right)$ & 1.052 & $0.585-1.894$ & 0.865 & & & \\
\hline ALB $(<35 \mathrm{~g} / \mathrm{L}$ versus $\geq 35 \mathrm{~g} / \mathrm{L})$ & 1.777 & $1.202-2.626$ & 0.004 & 1.387 & $0.911-2.111$ & 0.127 \\
\hline FIB $(>4 g / L$ versus $\leq 4 g / L)$ & 1.468 & $0.849-2.538$ & 0.169 & & & \\
\hline CEA $(>5 \mathrm{ng} / \mathrm{mL}$ versus $\leq 5 \mathrm{ng} / \mathrm{mL})$ & 1.104 & $0.773-1.575$ & 0.587 & & & \\
\hline CAI9-9 (>37U/mL versus $\leq 37 \mathrm{U} / \mathrm{mL})$ & 2.115 & $1.488-3.005$ & $<0.001$ & 1.313 & $0.83 \mathrm{I}-2.076$ & 0.243 \\
\hline CA72-4 $(>6.9 \mathrm{U} / \mathrm{mL}$ versus $\leq 6.9 \mathrm{U} / \mathrm{mL})$ & 1.798 & $1.216-2.659$ & 0.003 & 1.207 & $0.716-2.034$ & 0.480 \\
\hline $\operatorname{LMR}(<4.7 \mathrm{I}$ versus $\geq 4.7 \mathrm{I})$ & 0.777 & $0.566-1.068$ & 0.120 & & & \\
\hline NLR $(\geq 1.90$ versus $<1.90)$ & 1.247 & $0.920-1.689$ & 0.155 & & & \\
\hline PLR $(\geq 134.46$ versus $<134.46)$ & 1.322 & $0.974-1.793$ & 0.073 & & & \\
\hline AFR $(\geq 16.07$ versus $<16.07)$ & 1.729 & $1.145-2.611$ & 0.009 & 1.778 & $1.122-2.818$ & 0.014 \\
\hline $\operatorname{SLR}(\geq 1.28$ versus $<1.28)$ & 1.782 & $1.306-2.432$ & $<0.001$ & 1.512 & $1.058-2.161$ & 0.023 \\
\hline
\end{tabular}

Note: The $P$ values in bold font indicate statistical significance.

Abbreviations: HR, hazard ratio; $\mathrm{Cl}$, confidence interval; $\mathrm{ACT}$, adjuvant chemotherapy; $\mathrm{ADC}$, adenocarcinoma; SCC, squamous cell carcinoma; MANEC, mixed adenoneuroendocrine carcinoma; HGB, Hemoglobin; PLT, Platelets; ALB, albumin; FIB, fibrinogen; CEA, carcinoembryonic antigen; CA, carbohydrate antigen; LMR, lymphocyte-monocyte ratio; NLR, neutrophil-lymphocyte ratio; PLR, platelet-lymphocyte ratio; AFR, albumin-fibrinogen ratio; SLR, aspartate aminotransferase (AST)alanine aminotransferase (ALT) ratio. 
Table 3 Univariate and Multivariate Cox Regression Analyses of Prognostic Factors for Disease-Free Survival

\begin{tabular}{|c|c|c|c|c|c|c|}
\hline \multirow[t]{2}{*}{ Variables } & \multicolumn{3}{|c|}{ Univariate Analysis } & \multicolumn{3}{|c|}{ Multivariate Analysis } \\
\hline & HR & $95 \% \mathrm{Cl}$ & $\mathbf{P}$ & HR & $95 \% \mathrm{Cl}$ & $\mathbf{P}$ \\
\hline Age $(>60$ versus $\leq 60)$ & 2.094 & $1.630-2.689$ & $<0.001$ & $1.65 \mathrm{I}$ & $1.252-2.178$ & $<0.001$ \\
\hline Sex (male versus female) & 0.897 & $0.678-1.187$ & 0.447 & & & \\
\hline Family history (yes versus no) & 0.863 & $0.638-1.167$ & 0.339 & & & \\
\hline Diabetes (yes versus no) & 0.695 & $0.405-1.192$ & 0.187 & & & \\
\hline Hypertension (yes versus no) & 0.869 & $0.622-1.215$ & 0.412 & & & \\
\hline Thrombus (yes versus no) & 0.863 & $0.520-1.433$ & 0.569 & & & \\
\hline Tobacco (yes versus no) & 1.042 & $0.795-1.365$ & 0.765 & & & \\
\hline Alcohol (yes versus no) & 0.921 & $0.684-1.240$ & 0.588 & & & \\
\hline Blood type & & & 0.107 & & & \\
\hline Type A & 1 & & & & & \\
\hline Type B & 1.110 & $0.807-1.526$ & 0.523 & & & \\
\hline Type AB & 0.859 & $0.602-1.226$ & 0.402 & & & \\
\hline Type $\bigcirc$ & 1.426 & $0.949-2.144$ & 0.088 & & & \\
\hline ACT regimen & & & $<0.001$ & & & $<0.001$ \\
\hline Platinum-based doublet regimen & 1 & & & 1 & & \\
\hline Taxanes contained regimen & 0.171 & $0.100-0.292$ & $<0.001$ & 0.215 & $0.121-0.384$ & $<0.001$ \\
\hline Monotherapy & 0.160 & $0.087-0.293$ & $<0.001$ & 0.245 & $0.129-0.463$ & $<0.001$ \\
\hline Anthracycline-based regimen & 0.231 & $0.116-0.461$ & $<0.001$ & 0.180 & $0.084-0.386$ & $<0.001$ \\
\hline ACT cycles $(<5$ versus $\geq 5)$ & 2.328 & $1.791-3.026$ & $<0.001$ & 1.917 & $1.445-2.543$ & $<0.001$ \\
\hline Tumor location & & & 0.994 & & & \\
\hline Upper I/3 & $\mathrm{I}$ & & & & & \\
\hline Middle $1 / 3$ & 0.927 & $0.531-1.619$ & 0.791 & & & \\
\hline Lower $1 / 3$ & 0.935 & $0.515-1.696$ & 0.824 & & & \\
\hline Mix & 0.920 & $0.521-1.626$ & 0.774 & & & \\
\hline Tumor size $(\geq 4.5 \mathrm{~cm}$ versus $<4.5 \mathrm{~cm})$ & 1.813 & $1.411-2.330$ & $<0.001$ & 1.842 & I.397-2.429 & $<0.001$ \\
\hline Differentiation (poor versus high) & 2.054 & $1.519-2.776$ & $<0.001$ & 1.678 & $1.224-2.300$ & 0.001 \\
\hline Depth of invasion (T3-4 versus TI-2) & 1.825 & $1.368-2.434$ & $<0.001$ & 2.449 & $1.738-3.450$ & $<0.001$ \\
\hline Lymph node invasion (NI-3 versus N0) & 1.355 & $1.043-1.760$ & 0.023 & 2.000 & I.456-2.748 & $<0.001$ \\
\hline Pathology & & & 0.139 & & & \\
\hline ADC & I & & & & & \\
\hline $\mathrm{SCC}$ & 0.451 & $0.167-1.215$ & 0.115 & & & \\
\hline MANEC & 0.754 & $0.212-2.679$ & 0.662 & & & \\
\hline HER-2 (positive versus negative) & 1.818 & $1.33 \mathrm{I}-2.48 \mathrm{I}$ & $<0.001$ & 1.809 & $|.3| 3-2.49 \mid$ & $<0.001$ \\
\hline HGB $(<110 / 120 \mathrm{~g} / \mathrm{L}$ versus $\geq 110 / 120 \mathrm{~g} / \mathrm{L})$ & 1.475 & $1.143-1.904$ & 0.003 & 1.080 & $0.819-1.423$ & 0.585 \\
\hline PLT $\left(\geq 350 \times 10^{9} / \mathrm{L}\right.$ versus $\left.<350 \times 10^{9} / \mathrm{L}\right)$ & 0.670 & $0.375-1.196$ & 0.176 & & & \\
\hline ALB $(<35 g / L$ versus $\geq 35 g / L)$ & 1.713 & $1.250-2.348$ & 0.001 & $1.54 \mathrm{I}$ & $1.105-2.151$ & $0.01 \mathrm{I}$ \\
\hline FIB $(>4 g / L$ versus $\leq 4 g / L)$ & 1.474 & $0.943-2.306$ & 0.089 & & & \\
\hline CEA $(>5 \mathrm{ng} / \mathrm{mL}$ versus $\leq 5 \mathrm{ng} / \mathrm{mL})$ & 1.116 & $0.835-1.492$ & 0.459 & & & \\
\hline CA19-9 (>37U/mL versus $\leq 37 \mathrm{U} / \mathrm{mL})$ & 1.845 & $1.370-2.483$ & $<0.001$ & 1.354 & $0.959-1.913$ & 0.085 \\
\hline CA72-4 $(>6.9 \mathrm{U} / \mathrm{mL}$ versus $\leq 6.9 \mathrm{U} / \mathrm{mL})$ & 1.628 & I. $17 \mid-2.264$ & 0.004 & 1.101 & $0.738-1.642$ & 0.638 \\
\hline $\operatorname{LMR}(<4.7 \mathrm{I}$ versus $\geq 4.7 \mathrm{I})$ & 0.840 & $0.646-1.093$ & 0.194 & & & \\
\hline NLR $(\geq 1.90$ versus $<1.90)$ & I.187 & $0.926-1.522$ & 0.177 & & & \\
\hline PLR $(\geq 134.46$ versus $<134.46)$ & 1.157 & $0.902-1.483$ & 0.251 & & & \\
\hline AFR $(\geq 16.07$ versus $<16.07)$ & 1.413 & $0.984-2.029$ & 0.061 & 1.319 & $0.892-1.950$ & 0.165 \\
\hline SLR $(\geq 1.28$ versus $<1.28)$ & 1.417 & $1.103-1.819$ & 0.006 & 1.136 & $0.863-1.496$ & 0.364 \\
\hline
\end{tabular}

Note: The $P$ values in bold font indicate statistical significance.

Abbreviations: $\mathrm{HR}$, hazard ratio; $\mathrm{Cl}$, confidence interval; $\mathrm{ACT}$, adjuvant chemotherapy; $\mathrm{ADC}$, adenocarcinoma; SCC, squamous cell carcinoma; MANEC, mixed adenoneuroendocrine carcinoma; HGB, Hemoglobin; PLT, Platelets; ALB, albumin; FIB, fibrinogen; CEA, carcinoembryonic antigen; CA, carbohydrate antigen; LMR, lymphocyte-monocyte ratio; NLR, neutrophil-lymphocyte ratio; PLR, platelet-lymphocyte ratio; AFR, albumin-fibrinogen ratio; SLR, aspartate aminotransferase (AST)alanine aminotransferase (ALT) ratio. 
A

Points

Age, years

Blood type

Tumor size, cm

Tumor differentiation

Depth of invasion

Lymph node invasion

AFR

SLR

HER-2

Total Points

3-Year CSS

5-Year CSS

B

Points

Age, years

Tumor size, $\mathrm{cm}$

Tumor differentiation

Depth of invasion

Lymph node invasion

ALB, g/L

HER-2

Total Points

3-Year DFS

5-Year DFS
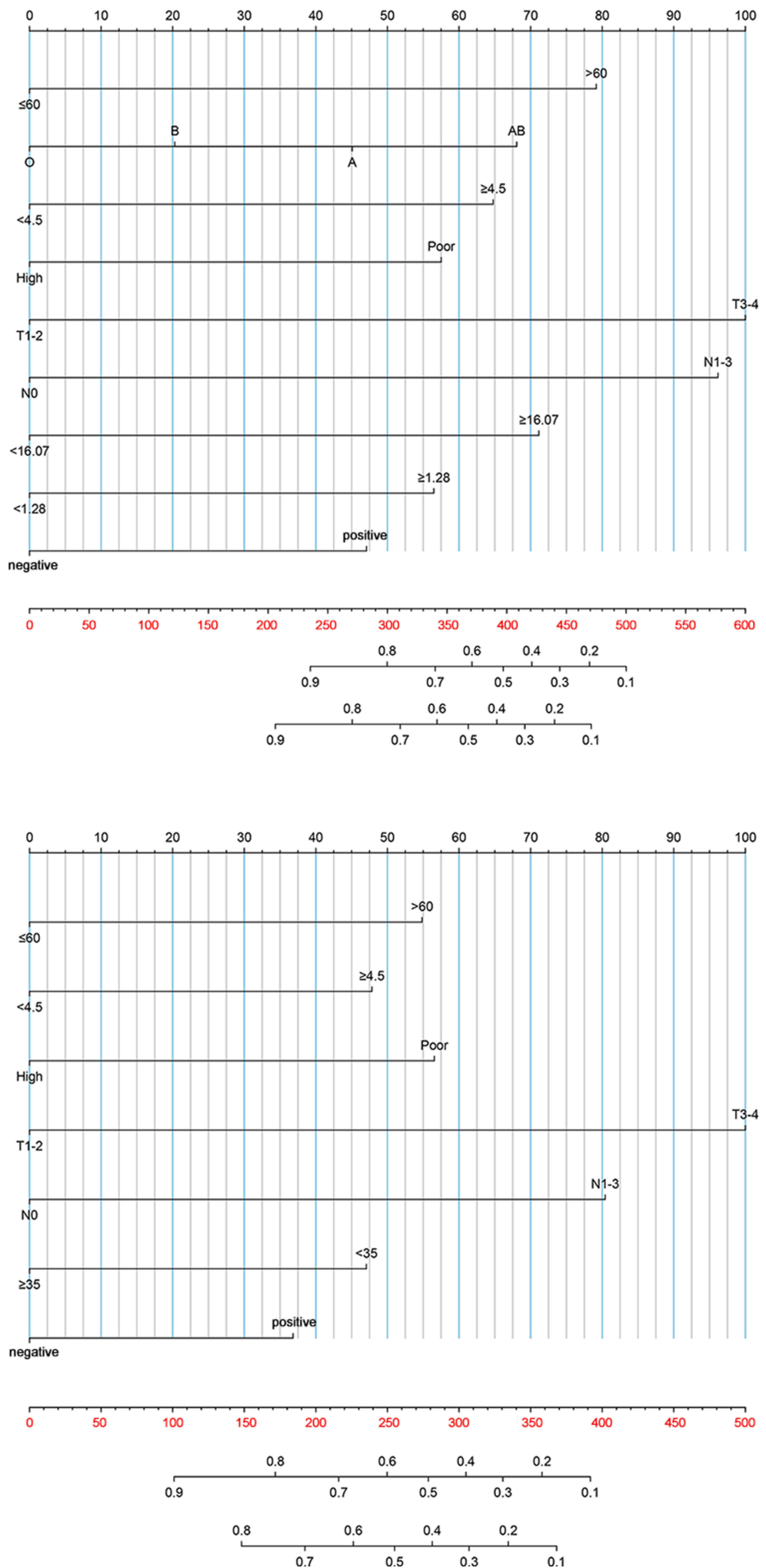

Figure I Nomograms predicting 3-year and 5-year cancer-specific survival (A) and disease-free survival (B) of stage II/III GC patients after curative gastrectomy followed by adjuvant chemotherapy.

Abbreviations: ACT, adjuvant chemotherapy; ALB, albumin; FIB, fibrinogen; AFR, albumin-fibrinogen ratio; SLR, aspartate aminotransferase (AST)-alanine aminotransferase (ALT) ratio. 
A

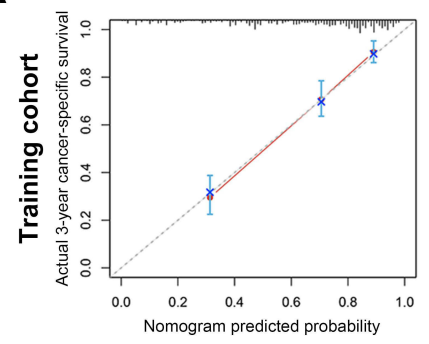

$\mathbf{E}$

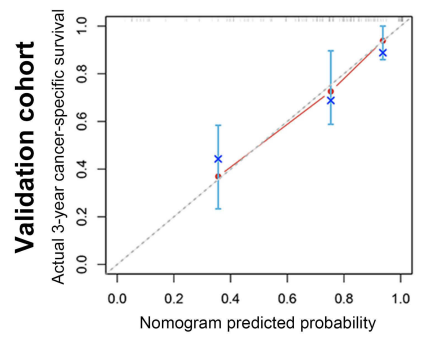

B

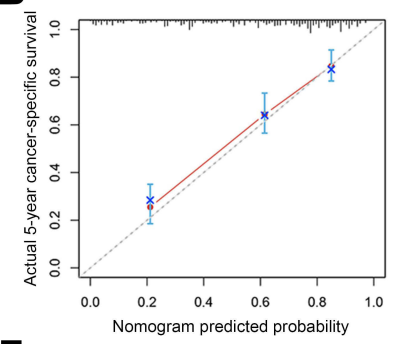

$\mathbf{F}$

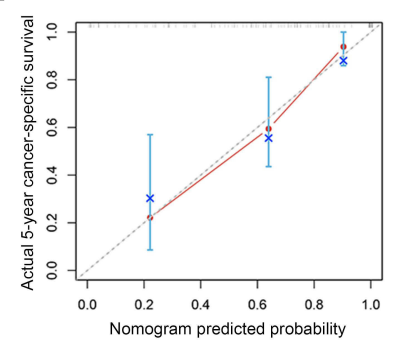

C

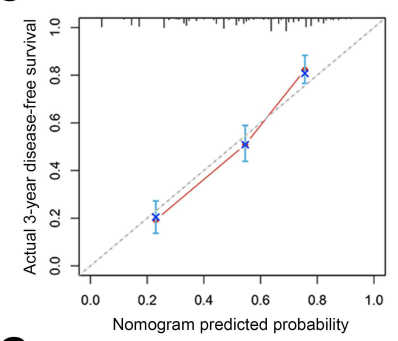

G

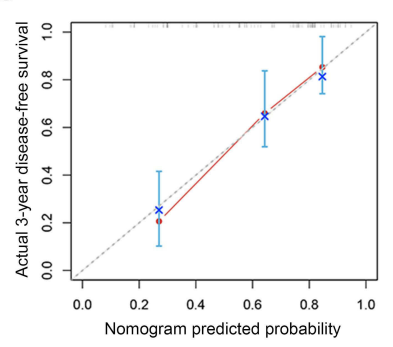

D

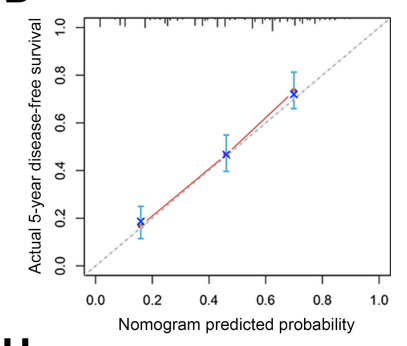

H

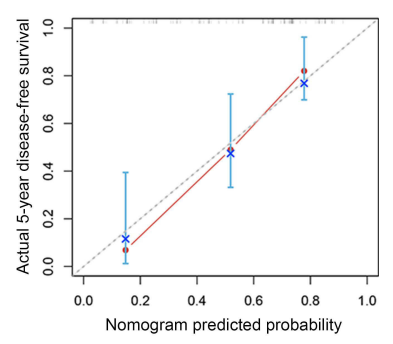

Figure 2 Calibration curves of nomogram for predicting 3-year cancer-specific survival (A), 5-year cancer-specific survival (B), 3-year disease-free survival (C), and 5-year disease-free survival (D) in the training cohort. Calibration curves of nomogram for predicting 3-year cancer-specific survival (E), 5-year cancer-specific survival (F), 3-year disease-free survival $(\mathbf{G})$, and 5 -year disease-free survival $(\mathbf{H})$ in the external validation cohort.

\section{Heat Maps and Survival Curves Based on the Nomograms}

The heat maps were created to observe the relationship between the combination of age and adjuvant chemotherapy cycles and survival probability based on the nomograms (Figure 3). This could visualize the correlation between the differences in independent predictors and 3-, and 5-year CSS and DFS. The blue area indicated a low survival rate, while the red area indicated a relatively good survival outcome. In addition, the risk scores in nomograms were the sum of each feature after weighted according to the cox proportional hazards models, and the formulas were as follows: risk score $(\mathrm{CSS})=0.923$ (if age $>60$ years old) +0.525 (if blood type was A) +0.237 (if blood type was B) +0.792 (if blood type was $\mathrm{AB}$ ) +0.755 (if tumor size $\geq 4.5 \mathrm{~cm}$ ) +0.670 (if tumor differentiation was poor) + 1.165 (if depth of invasion was T3-4) + 1.121 (if lymph node invasion was N1-3) +0.829 (if AFR $\geq 16.07$ ) +0.658 (if $\mathrm{SLR} \geq 1.28$ ) +0.549 (if HER-2 was positive); risk score (DFS) $=0.582$ (if age $>60$ years old) +0.507 (if tumor size $\geq 4.5 \mathrm{~cm}$ ) +0.600 (if tumor differentiation was poor) +1.061 (if depth of invasion was T3-4) +0.853 (if lymph node invasion was $\mathrm{N} 1-3$ ) +0.499 (if $\mathrm{ALB}<35 \mathrm{~g} / \mathrm{L}$ ) +0.390 (if HER-2 was positive). Other clinical features not mentioned in the above formulas were not allowed to be scored. The median risk scores were used as cut-off values to divide the training and validation cohorts into low-risk (CSS: $\leq 3.457$, DFS: $\leq 2.534$ ) and high-risk groups (CSS: >3.457, DFS: $>2.534$ ) in terms of CSS and DFS. Patients in the low-risk group had better survival than those in the high-risk group (Figure 4).

\section{Discussion}

In this study, we developed nomograms and corresponding heat maps to evaluate the CSS and DFS of stage II/III GC patients who underwent curative gastrectomy with adjuvant chemotherapy. The research results showed that the adjuvant chemotherapy cycles had important contributions to the survival rate of stage II/III GC, and they were of great significance for optimizing reasonable postoperative adjuvant treatment. We also successfully divided patients into lowrisk and high-risk groups based on the risk scores obtained, which helped to identify high-risk patients with poor prognosis.

As we know, systemic inflammation caused by complex host-tumor interaction is an important part of tumors, and plays a key role in inducing malignant tumors and determining disease progression. ${ }^{14,15}$ Persistent systemic inflammatory response can induce genetic instability and epigenetic modification directly through cytokine signal transduction or indirectly through the production of reactive oxygen and nitrogen species, leading to the occurrence of cancer. ${ }^{16,17}$ For 
A

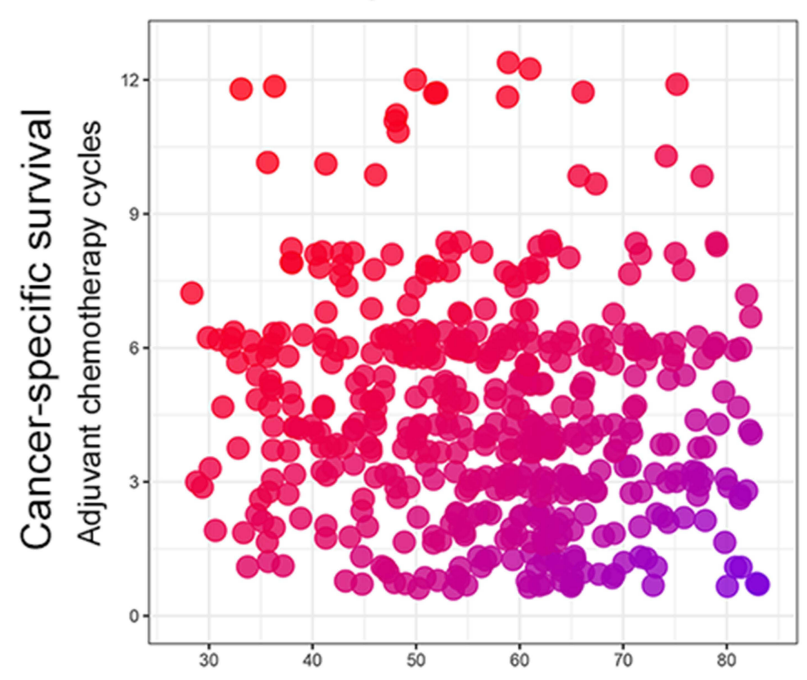

C

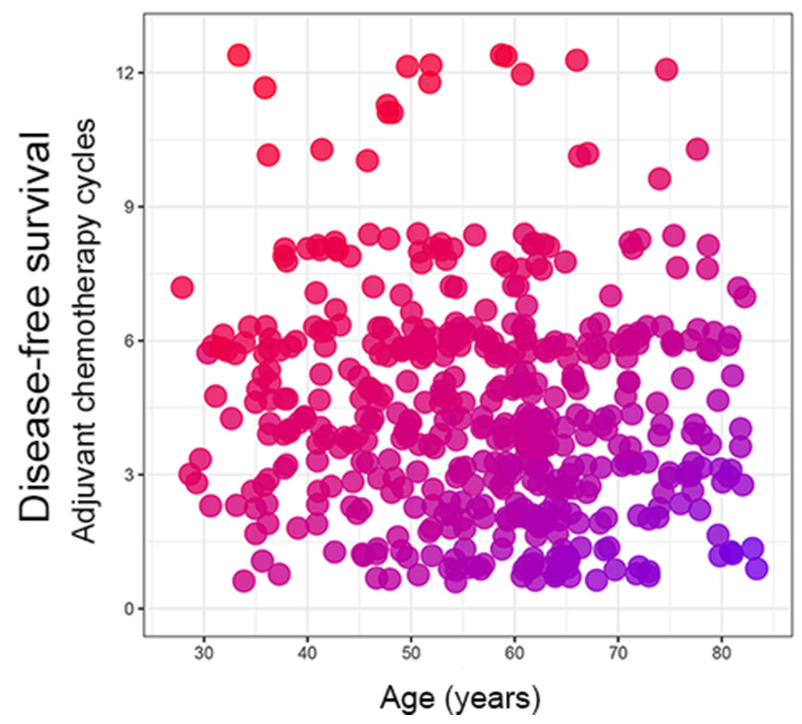

B

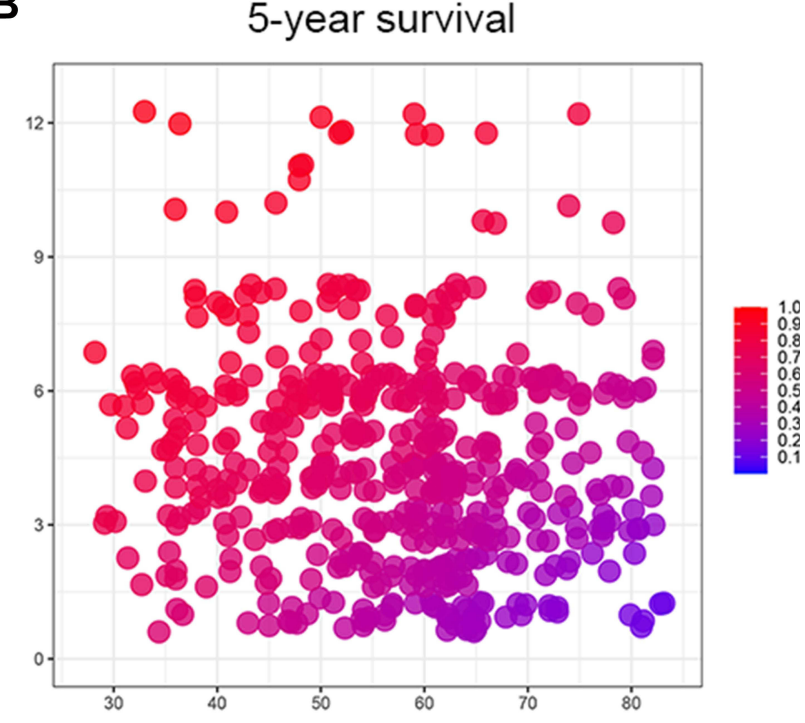

D

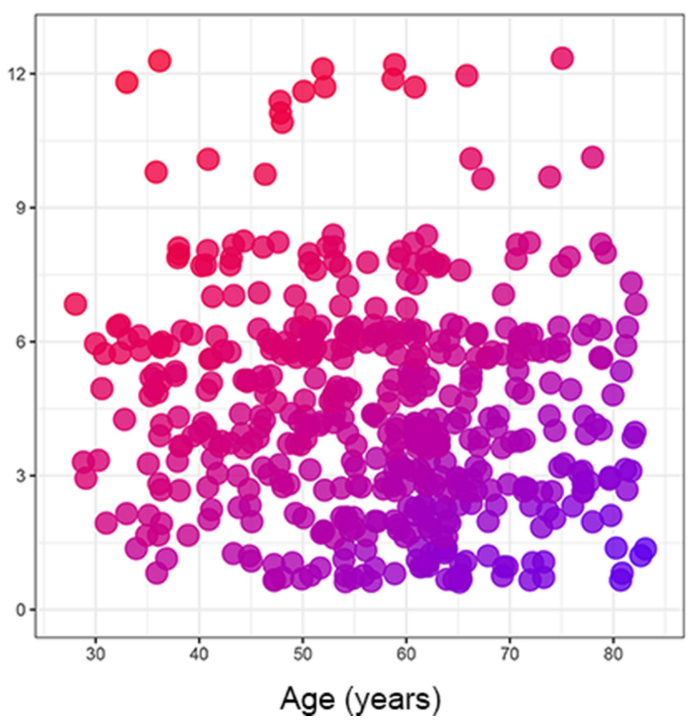

Figure 3 Heat maps of tumor age (x-axis) and adjuvant chemotherapy cycles ( $y$-axis) corresponding to 3-year cancer-specific survival (A), 5-year cancer-specific survival (B), 3-year disease-free survival (C), and 5-year disease-free survival (D).

Note: Red regions indicate relatively better outcomes than blue does.

example, pro-inflammatory cytokines, including IL-1 $\beta$, IL-6 and TNF- $\alpha$, directly or indirectly stimulate tumor proliferation and angiogenesis. ${ }^{14,17,18}$ In addition, the interaction between tumor cells and inflammation gradually produces a tumor microenvironment that promotes tumor angiogenesis and immunosuppression, and ultimately causes tumor growth, invasion and metastasis. ${ }^{14}$ Patients with systemic inflammation have a $50 \%$ lower survival rate than those without. ${ }^{19,20}$ Some previous studies have shown that many inflammatory indicators are related to the prognosis of GC, including LMR, NLR, PLR, SLR, etc., but their ability to predict survival are still controversial and requires further study. ${ }^{7,8,11,21}$

In addition, there are reports that nutritional status is closely related to the survival rate of cancer patients. ${ }^{22}$ Cancerrelated malnutrition is a complex multi-factor syndrome, which ultimately leads to poor treatment effects, postoperative complications and poor quality of life through weakened immune function and activation of systemic inflammation. ${ }^{23,24}$ Currently, ALB level is one of the most widely used indicators of nutritional status. The mechanism of hypoalbuminemia is related to the increase of two inflammatory cytokines, TNF- $\alpha$ and IL-6, which can inhibit the synthesis of ALB. ${ }^{25}$ Studies have shown that hypoalbuminemia is related to cancer progression and poor survival outcomes, which is related 
A

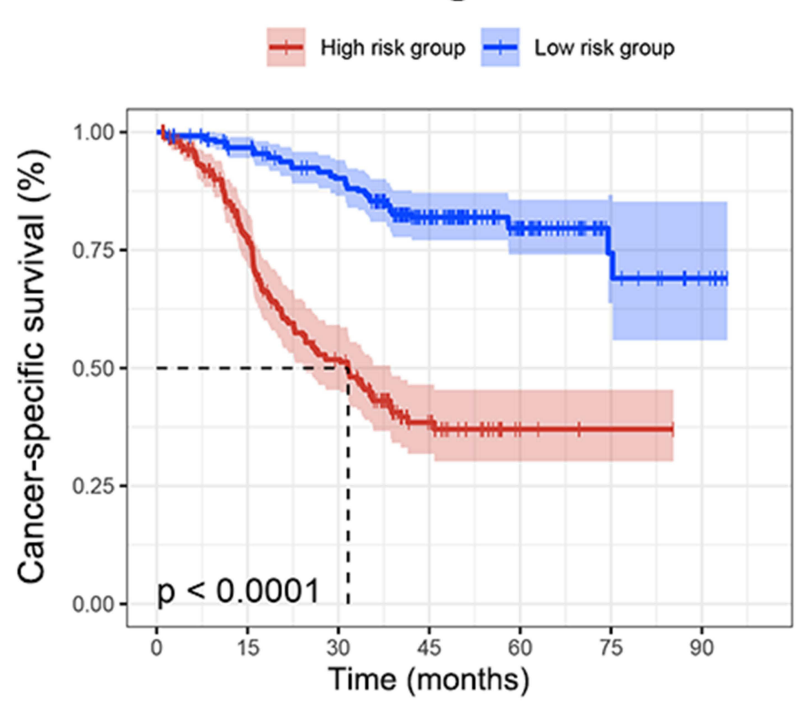

Number at risk

\begin{tabular}{|c|c|c|c|c|c|c|c|} 
High & 251 & 159 & 99 & 30 & 5 & 1 & 0 \\
Low & 252 & 229 & 206 & 131 & 60 & 14 & 5 \\
\hline
\end{tabular}

C

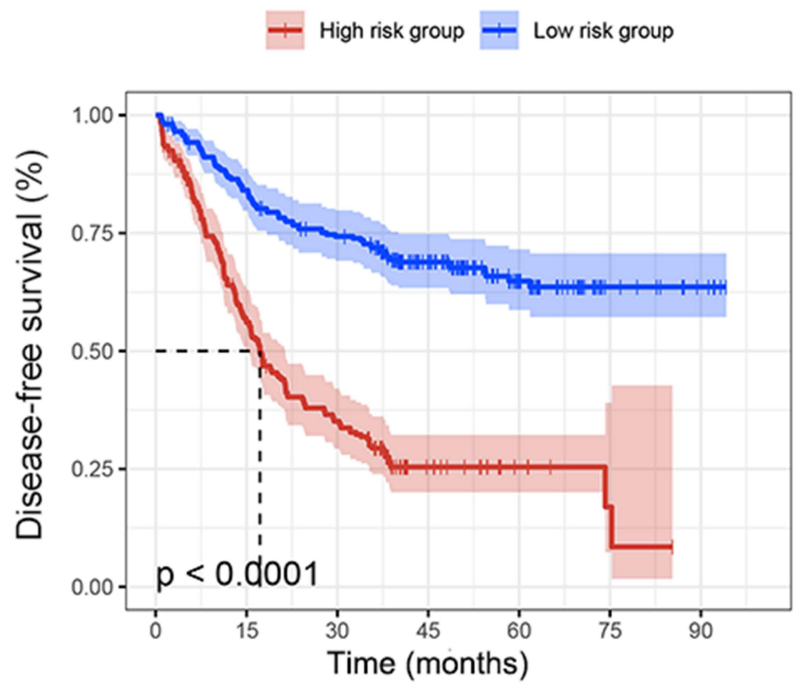

Number at risk

\begin{tabular}{c|c|c|c|c|c|c|c|}
\hline High & 241 & 121 & 75 & 19 & 6 & 2 & 0 \\
Low & 262 & 216 & 187 & 125 & 56 & 13 & 5 \\
\hline
\end{tabular}
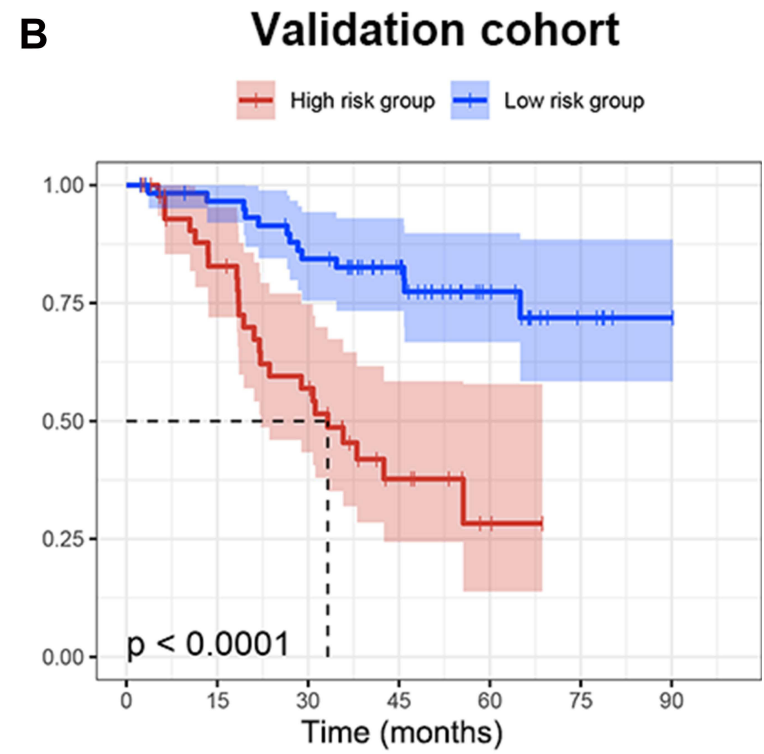

Number at risk

\begin{tabular}{c|c|c|c|c|c|c|c|}
\hline High & 47 & 33 & 22 & 8 & 2 & 0 & 0 \\
Low & 61 & 56 & 48 & 36 & 16 & 5 & 1 \\
\hline
\end{tabular}

D

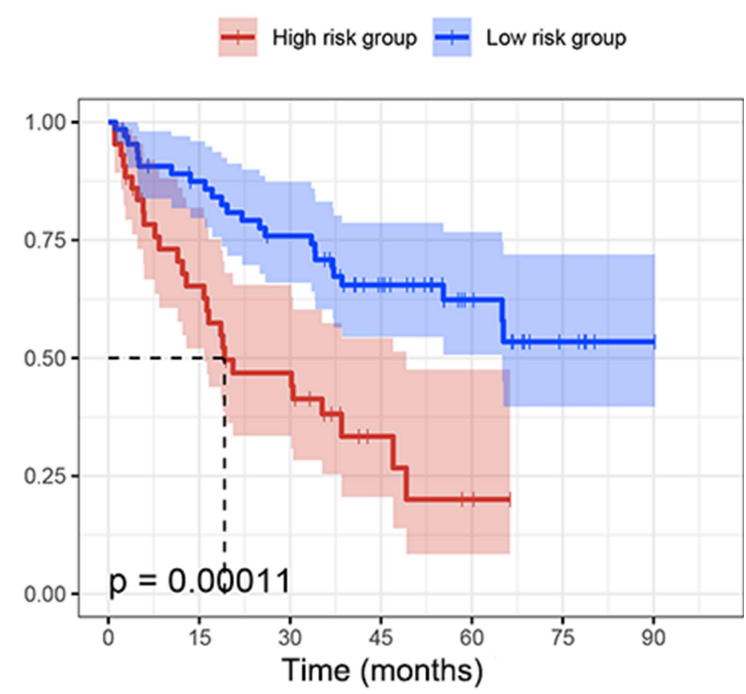

Number at risk

\begin{tabular}{|c|c|c|c|c|c|c|c|}
\hline High & 43 & 25 & 17 & 5 & 2 & 0 & 0 \\
Low & 65 & 53 & 45 & 31 & 15 & 5 & 1 \\
\hline
\end{tabular}

Figure 4 Kaplan-Meier survival curves of cancer-specific survival $(\mathbf{A})$ and disease-free survival $(\mathbf{C})$ in the training cohort based on risk scores. Kaplan-Meier survival curves of cancer-specific survival (B) and disease-free survival (D) in the external validation cohort based on risk scores.

to cachexia caused by chronic inflammation that consumes nutrition and energy in cancer patients. ${ }^{26}$ Hypoalbuminemia and systemic inflammation can promote the synthesis of fibrinogen. ${ }^{18,27}$ Fibrinogen can form a physical barrier with platelets to prevent natural killer cells from contacting tumor cells, thereby increasing the survival rate of circulating tumor cells and promoting tumor metastasis potential. ${ }^{28}$

As a new and effective tool, the clinical prognosis nomogram can quantify individual risks and predict survival outcomes. More and more studies try to use clinical prognosis scoring model instead of TNM staging to predict the 
survival rate of patients, including esophageal cancer, ${ }^{29}$ colorectal cancer, ${ }^{30}$ prostate cancer $^{31}$ and seminoma. ${ }^{32}$ At the present study, we have established nomograms to display which factors are related to the prognosis of stage II/III GC with adjuvant chemotherapy, and to identify patients with poor prognosis in time and provide close follow-up. In the final nomograms, age, depth of invasion, lymph node invasion, tumor size, tumor differentiation and HER-2 were independent risk factors common to the CSS and DFS of patients. The blood type, AFR, and SLR were the unique predictors of CSS, and ALB was included in the predictors of DFS. The difference in indicators between CSS and DFS might be due to the fact that CSS, as a survival indicator based on death outcome, emphasized the interaction of various systems throughout the body. Whether it was a training cohort or an external validation cohort, the calibration curve showed that the prediction result of our model was in good agreement with the actual situation. At the same time, the C-index performed well in both the training cohort (CSS: 0.774, DFS: 0.713) and the validation cohort (CSS: 0.788, DFS: 0.727). These results indicated that our clinical nomograms performed well in predicting survival.

Some studies have confirmed that neoadjuvant chemotherapy can reduce the recurrence rate of tumors and increase the cure rate of complete tumor resection. ${ }^{33,34}$ However, this study focused on the efficacy of adjuvant chemotherapy and excluded patients who received neoadjuvant chemotherapy, so further study was needed to determine its benefit in such patients. In fact, although D2 gastrectomy is a globally recognized surgical standard, its optimal adjuvant treatment has not yet been determined. ${ }^{35}$ For example, in Japan, single-dose postoperative chemotherapy with oral fluoropyrimidine S-1 was a standard selection based on the results of the ACTS-GC trial. ${ }^{36}$ In Europe, they preferred postoperative chemotherapy with epirubicin, cisplatin, and 5-fluorouracil based on evidence from MAGIC trial. ${ }^{34}$ These inconsistencies could not completely rule out the differences in tumor biology of different races. But it was certain that a study by Bang et al showed that dual combination chemotherapy including platinum compounds after D2 surgery in Asian stage II/III GC patients was effective for GC. ${ }^{37}$ Targeted therapy and immunotherapy have developed rapidly in recent years, and HER-2 has been routinely tested as a representative targeted biomarker. ${ }^{38,39}$ Our study data suggest that positive HER-2 was a risk factor for GC prognosis, which was the same as the findings of most scholars. ${ }^{40,41}$ The amplification of HER-2 gene or abnormal protein expression predicted the recurrence of GC, invasion and metastasis of tumor cells and reduced survival rate of patients. ${ }^{40,42}$ These evidences strongly confirm that HER-2 was a key target in the antiinvasion therapy of GC, and provided a certain basis for the study of HER-2-positive GC targeted drugs such as trastuzumab. ${ }^{43,44}$

Our research results also showed that the adjuvant chemotherapy cycles were closely related to the survival of GC patients. Properly prolonging the chemotherapy cycles could achieve a better survival prognosis. Therefore, we established heat maps of the combination of age and adjuvant chemotherapy cycles based on the Cox regression models and the nomograms. Heat maps could be observed that even patients of the same age range had different treatment cycles, which was inextricably linked to individual patient tolerance, compliance, financial status and doctoral decision-making. At the same time, we also found that the survival rate of patients over 60 years who underwent low-cycle adjuvant chemotherapy decreased significantly. With the increase of patients' age, the survival benefit was gradually insignificant. The treatment advices given by clinicians according to the individual situation of patients can effectively reduce the risk of excessive adjuvant chemotherapy. In addition, we divided patients into low-risk and high-risk groups with significant differences based on the risk stratification established by risk scores. Patients in the high-risk group generally had poorer survival rate, and adjuvant chemotherapy might provide them with a stronger survival benefit. This also provided inspiration for clinical decision-making, making treatment more individualized.

However, we acknowledge that this study has some limitations. First of all, it was a retrospective study, so future prospective studies are needed to validate our nomograms. Secondly, the research subjects we included were all Chinese, and we need to further incorporate information of different races to evaluate the models. Finally, we failed to include some potential predictors because they were not routinely checked, such as helicobacter pylori infection, PG I/II, EGFR mutations, etc. 


\section{Conclusion}

In conclusion, this study established and verified a nomogram that could effectively predict the CSS and DFS of stage II/ III GC patients who underwent curative gastrectomy with adjuvant chemotherapy and innovatively applied heat map visualization predictors. It could help clinicians to more accurately assess the survival status of patients and further promote personalized treatment, which had important clinical practicability.

\section{Acknowledgments}

We thank all medical staff who have contributed to the medical record system, as well as the editors and reviewers for their valuable suggestions on this manuscript.

\section{Disclosure}

The authors declare that the research was conducted in the absence of any commercial or financial relationships that could be construed as a potential conflict of interest.

\section{References}

1. Sitarz R, Skierucha M, Mielko J, Offerhaus GJA, Maciejewski R, Polkowski WP. Gastric cancer: epidemiology, prevention, classification, and treatment. Cancer Manag Res. 2018;10:239-248. doi:10.2147/CMAR.S149619

2. Balakrishnan M, George R, Sharma A, Graham DY. Changing trends in stomach cancer throughout the world. Curr Gastroenterol Rep. 2017;19 (8):36. doi:10.1007/s11894-017-0575-8

3. Van Cutsem E, Sagaert X, Topal B, Haustermans K, Prenen H. Gastric cancer. Lancet. 2016;388(10060):2654-2664. doi:10.1016/S0140-6736(16) 30354-3

4. Gambardella V, Cervantes A. Precision medicine in the adjuvant treatment of gastric cancer. Lancet Oncol. 2018;19(5):583-584. doi:10.1016/ S1470-2045(18)30131-1

5. Kaytan Saglam E, Yucel S, Balik E, et al. Adjuvant chemoradiotherapy after D2 resection in gastric cancer: a single-center observational study. J Cancer Res Clin Oncol. 2015;141(2):361-367. doi:10.1007/s00432-014-1816-y

6. Jagadesham VP, Lagarde SM, Immanuel A, Griffin SM. Systemic inflammatory markers and outcome in patients with locally advanced adenocarcinoma of the oesophagus and gastro-oesophageal junction. Br J Surg. 2017;104(4):401-407. doi:10.1002/bjs.10425

7. Liu X, Wu Z, Lin E, et al. Systemic prognostic score and nomogram based on inflammatory, nutritional and tumor markers predict cancer-specific survival in stage II-III gastric cancer patients with adjuvant chemotherapy. Clin Nutr. 2019;38(4):1853-1860. doi:10.1016/j.clnu.2018.07.015

8. Qu JL, Qu XJ, Li Z, et al. Prognostic model based on systemic inflammatory response and clinicopathological factors to predict outcome of patients with node-negative gastric cancer. PLoS One. 2015;10(6):e0128540. doi:10.1371/journal.pone.0128540

9. Li SQ, Jiang YH, Lin J, et al. Albumin-to-fibrinogen ratio as a promising biomarker to predict clinical outcome of non-small cell lung cancer individuals. Cancer Med. 2018;7(4):1221-1231. doi:10.1002/cam4.1428

10. Gao QF, Qiu JC, Huang XH, et al. The predictive and prognostic role of a novel ADS score in esophageal squamous cell carcinoma patients undergoing esophagectomy. Cancer Cell Int. 2018;18:153. doi:10.1186/s12935-018-0648-2

11. Li L, Zeng Q, Xue N, et al. A nomogram based on aspartate aminotransferase/alanine aminotransferase (AST/ALT) ratio to predict prognosis after surgery in gastric cancer patients. Cancer Control. 2020;27(1):1073274820954458. doi:10.1177/1073274820954458

12. Han DS, Suh YS, Kong SH, et al. Nomogram predicting long-term survival after d2 gastrectomy for gastric cancer. J Clin Oncol. 2012;30 (31):3834-3840. doi:10.1200/JCO.2012.41.8343

13. Jiang Y, Li T, Liang X, et al. Association of adjuvant chemotherapy with survival in patients with stage II or III gastric cancer. JAMA Surg. 2017;152(7):e171087. doi:10.1001/jamasurg.2017.1087

14. Mantovani A, Allavena P, Sica A, Balkwill F. Cancer-related inflammation. Nature. 2008;454(7203):436-444. doi:10.1038/nature07205

15. Diakos CI, Charles KA, McMillan DC, Clarke SJ. Cancer-related inflammation and treatment effectiveness. Lancet Oncol. 2014;15(11):e493-503. doi:10.1016/S1470-2045(14)70263-3

16. Colotta F, Allavena P, Sica A, Garlanda C, Mantovani A. Cancer-related inflammation, the seventh hallmark of cancer: links to genetic instability. Carcinogenesis. 2009;30(7):1073-1081. doi:10.1093/carcin/bgp127

17. Grivennikov SI, Greten FR, Karin M. Immunity, inflammation, and cancer. Cell. 2010;140(6):883-899. doi:10.1016/j.cell.2010.01.025

18. Balkwill F, Mantovani A. Inflammation and cancer: back to Virchow? Lancet. 2001;357(9255):539-545. doi:10.1016/S0140-6736(00)04046-0

19. Guthrie GJ, Charles KA, Roxburgh CS, Horgan PG, McMillan DC, Clarke SJ. The systemic inflammation-based neutrophil-lymphocyte ratio: experience in patients with cancer. Crit Rev Oncol Hematol. 2013;88(1):218-230. doi:10.1016/j.critrevonc.2013.03.010

20. McMillan DC. The systemic inflammation-based Glasgow prognostic score: a decade of experience in patients with cancer. Cancer Treat Rev. 2013;39(5):534-540. doi:10.1016/j.ctrv.2012.08.003

21. Shinko D, Diakos CI, Clarke SJ, Charles KA. Cancer-related systemic inflammation: the challenges and therapeutic opportunities for personalized medicine. Clin Pharmacol Ther. 2017;102(4):599-610. doi:10.1002/cpt.789

22. Mantzorou M, Koutelidakis A, Theocharis S, Giaginis C. Clinical value of nutritional status in cancer: what is its impact and how it affects disease progression and prognosis? Nutr Cancer. 2017;69(8):1151-1176. doi:10.1080/01635581.2017.1367947

23. Van Cutsem E, Arends J. The causes and consequences of cancer-associated malnutrition. Eur J Oncol Nurs. 2005;9(Suppl 2):S51-63. doi:10.1016/ j.ejon.2005.09.007

24. Ackerman D, Laszlo M, Provisor A, Yu A. Nutrition management for the head and neck cancer patient. Cancer Treat Res. 2018;174:187-208. 
25. Tanaka T, Narazaki M, Kishimoto T. IL-6 in inflammation, immunity, and disease. Cold Spring Harb Perspect Biol. 2014;6(10):a016295. doi:10.1101/cshperspect.a016295

26. McSorley ST, Watt DG, Horgan PG, McMillan DC. Postoperative systemic inflammatory response, complication severity, and survival following surgery for colorectal cancer. Ann Surg Oncol. 2016;23(9):2832-2840. doi:10.1245/s10434-016-5204-5

27. Dvorak HF. Tumors: wounds that do not heal-redux. Cancer Immunol Res. 2015;3(1):1-11. doi:10.1158/2326-6066.CIR-14-0209

28. Palumbo JS, Talmage KE, Massari JV, et al. Platelets and fibrin (ogen) increase metastatic potential by impeding natural killer cell-mediated elimination of tumor cells. Blood. 2005;105(1):178-185. doi:10.1182/blood-2004-06-2272

29. Semenkovich TR, Yan Y, Subramanian M, et al. A clinical nomogram for predicting node-positive disease in esophageal cancer. Ann Surg. 2021;273(6):e214-e221. doi:10.1097/SLA.0000000000003450

30. Sjoquist KM, Renfro LA, Simes RJ, et al. Personalizing survival predictions in advanced colorectal cancer: the ARCAD nomogram project. $J$ Natl Cancer Inst. 2018;110(6):638-648. doi:10.1093/jnci/djx253

31. Diamand R, Ploussard G, Roumiguié M, et al. External validation of a multiparametric magnetic resonance imaging-based nomogram for the prediction of extracapsular extension and seminal vesicle invasion in prostate cancer patients undergoing radical prostatectomy. Eur Urol. 2021;79 (2):180-185. doi:10.1016/j.eururo.2020.09.037

32. Aparicio J, Maroto P, García Del Muro X, et al. Prognostic factors for relapse in stage I seminoma: a new nomogram derived from three consecutive, risk-adapted studies from the Spanish Germ Cell Cancer Group (SGCCG). Ann Oncol. 2014;25(11):2173-2178. doi:10.1093/annonc/ mdu437

33. Al-Batran SE, Homann N, Pauligk C, et al. Perioperative chemotherapy with fluorouracil plus leucovorin, oxaliplatin, and docetaxel versus fluorouracil or capecitabine plus cisplatin and epirubicin for locally advanced, resectable gastric or gastro-oesophageal junction adenocarcinoma (FLOT4): a randomised, Phase 2/3 trial. Lancet. 2019;393(10184):1948-1957. doi:10.1016/S0140-6736(18)32557-1

34. Cunningham D, Allum WH, Stenning SP, et al. Perioperative chemotherapy versus surgery alone for resectable gastroesophageal cancer. $N$ Engl $J$ Med. 2006;355(1):11-20. doi:10.1056/NEJMoa055531

35. Okines A, Verheij M, Allum W, Cunningham D, Cervantes A. Gastric cancer: ESMO clinical practice guidelines for diagnosis, treatment and follow-up. Ann Oncol. 2010;21(Suppl 5):v50-54. doi:10.1093/annonc/mdq164

36. Sasako M, Sakuramoto S, Katai H, et al. Five-year outcomes of a randomized Phase III trial comparing adjuvant chemotherapy with S-1 versus surgery alone in stage II or III gastric cancer. J Clin Oncol. 2011;29(33):4387-4393. doi:10.1200/JCO.2011.36.5908

37. Bang YJ, Kim YW, Yang HK, et al. Adjuvant capecitabine and oxaliplatin for gastric cancer after D2 gastrectomy (CLASSIC): a Phase 3 open-label, randomised controlled trial. Lancet. 2012;379(9813):315-321. doi:10.1016/S0140-6736(11)61873-4

38. Lee J, Kim ST, Kim K, et al. Tumor genomic profiling guides patients with metastatic gastric cancer to targeted treatment: the viktory umbrella trial. Cancer Discov. 2019;9(10):1388-1405. doi:10.1158/2159-8290.CD-19-0442

39. Kim ST, Cristescu R, Bass AJ, et al. Comprehensive molecular characterization of clinical responses to PD-1 inhibition in metastatic gastric cancer. Nat Med. 2018;24(9):1449-1458. doi:10.1038/s41591-018-0101-Z

40. Lei YY, Huang JY, Zhao QR, et al. The clinicopathological parameters and prognostic significance of HER2 expression in gastric cancer patients: a meta-analysis of literature. World J Surg Oncol. 2017;15(1):68. doi:10.1186/s12957-017-1132-5

41. Park DI, Yun JW, Park JH, et al. HER-2/neu amplification is an independent prognostic factor in gastric cancer. Dig Dis Sci. 2006;51(8):1371-1379. doi:10.1007/s10620-005-9057-1

42. Dang HZ, Yu Y, Jiao SC. Prognosis of HER2 over-expressing gastric cancer patients with liver metastasis. World J Gastroenterol. 2012;18 (19):2402-2407. doi:10.3748/wjg.v18.i19.2402

43. Bang YJ, Van Cutsem E, Feyereislova A, et al. Trastuzumab in combination with chemotherapy versus chemotherapy alone for treatment of HER2-positive advanced gastric or gastro-oesophageal junction cancer (ToGA): a phase 3, open-label, randomised controlled trial. Lancet. 2010;376(9742):687-697. doi:10.1016/S0140-6736(10)61121-X

44. Shitara K, Bang YJ, Iwasa S, et al. Trastuzumab deruxtecan in previously treated her2-positive gastric cancer. $N$ Engl $J$ Med. 2020;382 (25):2419-2430. doi:10.1056/NEJMoa2004413

Cancer Management and Research

Dovepress

Publish your work in this journal

Cancer Management and Research is an international, peer-reviewed open access journal focusing on cancer research and the optimal use of preventative and integrated treatment interventions to achieve improved outcomes, enhanced survival and quality of life for the cancer patient. The manuscript management system is completely online and includes a very quick and fair peer-review system, which is all easy to use. Visit http://www.dovepress.com/testimonials.php to read real quotes from published authors.

Submit your manuscript here: https://www.dovepress.com/cancer-management-and-research-journal 